Bundesgesundheitsbl -

Gesundheitsforsch - Gesundheitsschutz

2000 • 43:683-693 $\odot$ Springer-Verlag 2000
Leitthema: AIDS

L.Voß

Robert Koch-Institut, Berlin

\section{Zusammenfassung}

Angesichts einer grundlegenden Neugestaltung der Surveillance übertragbarer Erkrankungen mit dem neuen Infektionsschutzgesetz, die u.a.von den Erfahrungen der HIVund AIDS-Strategie profitiert hat, wird in dieser Untersuchung ein spezieller Aspekt dieser Strategie beleuchtet. Das bundesdeutsche Gesamtkonzept zur Bekämpfung von HIV und AIDS wurde von den meisten Bundesländern weitestgehend übernommen. Einige Bundesländer schufen jedoch für spezielle Bereiche bzw. Bevölkerungsgruppen Sonderregelungen. Auch wenn allgemeine Testempfehlungen oder Routinetestungen auf HIV (außer bei Blutspendern) stets abgelehnt wurden, stellt sich die praktische Umsetzung einer im Grundsatz eher liberalen AIDS-Politik in einzelnen Bevölkerungsgruppen durchaus unterschiedlich dar. Besonders deutlich wird dies am Beispiel Justizvollzug bzw. Asylbewerber bzw. Ausländer. Im Zeitverlauf können in Anbetracht verbesserter therapeutischer Möglichkeiten Veränderungen in der Einstellung zum Test beobachtet werden.

\section{Schlüsselwörter}

AIDS-Politik · HIV-Antikörpertest · Routinetest - Testpolitik · Testpraxis · Freiwilligkeit

\title{
HIV-Testpolitik und -Testpraxis in Deutschland - eine Bestandsaufnahme
}

Z Jahresende wird das Infektionsschutzgesetz in Kraft treten; mit ihm werden das bisherige Bundesseuchengesetz, das Geschlechtskrankheitengesetz sowie einige weitere Verordnungen abgelöst. Dem im Wesentlichen aus dem Jahre 1961 stammenden Bundes-Seuchengesetz lag ein Konzept der Durchführung von Maßnahmen zur klassischen Seuchenabwehr zugrunde. Ihm fehlen jedoch weitestgehend moderne Instrumente zur Erkennung und Überwachung übertragbarer Krankheiten. So sind beispielsweise Informationsstrukturen für eine zentrale epidemiologische Auswertung nicht vorgesehen, die Voraussetzung dafür ist, Zusammenhänge schnell erkennen und zielgerichtet reagieren zu können. Für die HIV-Infektion, die weder im Katalog der nach dem Bundes-Seuchengesetz meldepflichtigen Krankheiten, noch im 1953 geschaffenen Geschlechtskrankheitengesetz enthalten ist, wurde 1987 die "Verordnung über die Berichtspflicht für positive HIV-Bestätigungstests (Laborberichtsverordnung)" [1] geschaffen. Nach dieser Regelung waren positive Ergebnisse beim Nachweis von Antikörpern gegen HIV anonym zu melden. Im neuen Infektionsschutzgesetz ist nun HIV als hauptsächlich sexuell übertragbarer Krankheitserreger neben Syphilis unter $\$ 7$, Abs. 3 aufgeführt. Die Meldung wird weiterhin aus epidemiologischen - nicht aus seuchenpolitischen - Gründen gefordert, deshalb soll sie auch künftig nicht namentlich erfolgen [2]. Neu ist jedoch die Angabe der aus dem AIDS-Fallregister bekannten und bewährten Namenskodierung, die zusammen mit den anderen im Gesetz vorgegebenen Angaben Doppelmeldungen mit hinreichend hoher Wahrscheinlichkeit erkennen lässt; eine Notwendigkeit, die im Hinblick auf eine valide epidemiologische Analyse unerlässlich ist. Diese Analysen sind um so wichtiger als die bisher verwendeten Daten aus dem freiwilligen AIDS-Fallregister im Zuge rückläufiger Zahlen aufgrund einer verlängerten Inkubationszeit durch effektivere Medikamente immer mehr an Bedeutung verlieren. Die veränderte HIVRegistrierung steht darüber hinaus im Einklang mit Empfehlungen des European Centre for Epidemiological Monitoring of AIDS, eine HIV-Fallerfassung $\mathrm{zu}$ installieren.

Nicht von ungefähr gilt das Auftreten von HIV/AIDS Anfang der achtziger Jahre als eine Zäsur in der Wahrnehmung und im Umgang mit Infektionserkrankungen in den modernen Industriegesellschaften. Am Beispiel HIV/ AIDS wurden exemplarisch neue Formen der epidemiologischen Surveillance, der gesellschaftlichen und individuellen Prävention und Verhaltensbeeinflussung sowie der Krankenversorgung erprobt und etabliert. Wie sind die ursprünglichen Intentionen verwirklicht worden? Wo haben sich traditionelle Herangehensweisen bewährt? In welchen Bereichen bleibt die Testpolitik widersprüchlich und inkonsistent?

Dr. Lieselotte Voß

Robert Koch-Institut, Postfach 650280 13302 Berlin 
Bundesgesundheitsbl -

Gesundheitsforsch - Gesundheitsschutz

2000 • 43:683-693 @ Springer-Verlag 2000

\section{L.Voß}

\section{HIV-testpolicy and -testpractice in Germany - An Inventory}

\section{Abstract}

The new "act on the prevention and control of infectious diseases in man" will fundamentally reform the surveillance of communicable diseases. This reformation has profited from the experiences of the HIV-and AIDS-strategy. A special aspect of this strategy is enlightened in this article. Germany's federal concept for fighting HIV and AIDS was accepted in most points by most of the Länder. However, some of them established special regulations for different fields and/or subpopulations. Although general test recommendations or routine tests for HIV-antibodies had been denied (except for blood donors), the implementation of a basically liberal AIDS-policy varied according to different subgroups. The treatment of prisoners, persons seeking political asylum or foreigners serve as enlightening examples. In the course of time the improvement of therapeutic possibilities led to changes in the attitude towards the test.

\section{Keywords}

AIDS-policy · HIV-antibodytest · Routinetest Testpolicy $\cdot$ Testpractice $\cdot$ Informed consent

Leitthema: AIDS

Vor dem Hintergrund einer grundlegenden Neugestaltung der Surveillance übertragbarer Erkrankungen soll hier am Beispiel HIV eine Bestandsaufnahme über die Testpolitik und -praxis in Deutschland versucht werden.

\section{Untersuchung auf Krank- heiten/Erreger allgemein}

In Deutschland werden Bevölkerungsteile generell nicht routinemäßig auf Infektionskrankheiten untersucht. Dies war weder im Bundes-Seuchengesetz vorgegeben, noch ist es im Rahmen des Infektionsschutzgesetzes vorgesehen. Es gibt jedoch Bereiche, in denen besondere Ermächtigungen zur Untersuchung von bestimmten Personengruppen bestehen; diese generellen Ermächtigungen werden allerdings in den Bundesländern sehr unterschiedlich gehandhabt.

$\mathrm{Zu}$ den genannten Personengruppen gehören Menschen, die sich in der Bundesrepublik um Asyl bewerben. Nach $\$ 4.1$ Asylverfahrensgesetz (1. Juli 1993) haben "Ausländer, die in einer Aufnahmeeinrichtung oder Gemeinschaftsunterkunft wohnen... eine ärztliche Untersuchung auf übertragbare Krankheiten einschließlich einer Röntgenaufnahme der Atmungsorgane zu dulden. Die Länder bestimmen den Umfang der Untersuchung ...”.

Ferner ermächtigt ein berufsgenossenschaftlicher Grundsatz (G42; der aus dem Jahre 1987 stammende Grundsatz wird z. Z. überarbeitet) [3] den Arzt, im Rahmen von arbeitsmedizinischen Vorsorgeuntersuchungen, gezielte mikrobiologische Testungen zum frühen Nachweis bzw. zur Verhinderung von Infektionskrankheiten durchzuführen. Menschen, die im Lebensmittelbereich beschäftigt werden, dürfen nach $\$ \$$ 17-18 Gesetz zur Verhütung und Bekämpfung übertragbarer Krankheiten beim Menschen (1961) erst dann ihre Tätigkeit aufnehmen, wenn eine Reihe von definierten Erkrankungen ausgeschlossen bzw. erwiesen wurde, dass sie nicht Ausscheider bestimmter, auf Lebensmittel übertragbarer Erreger sind.

\section{Routinetestungen auf HIV-Antikörper}

Die einzige gesetzlich geregelte Routinetestung ist die serologische Testung aller Blutspenden auf Infektionserreger, u. a. auf HIV $[4,5,6]$. Darüber hinaus wurde das Angebot eines freiwilligen HIVAntikörpertestes in die Mutterschaftsrichtlinien aufgenommen. Ereignisse, in deren Rahmen eine Routinetestung in Deutschland erneut zur Diskussion stand, waren - unter dem Hauptaspekt der Primärprävention - z. B. Meldungen über die vermutliche Infektion von sechs Patienten durch ihren Zahnarzt in Florida 1991 und der so genannte "Blutskandal" 1993. Im ersten Fall wurde in öffentlichen Diskussionen die Forderung nach regelmäßiger Testung des medizinischen Personals erhoben. Im zweiten Fall zog der Gesundheitsminister in Erwägung, bei jeder ärztlichen Blutabnahme - außer im Falle ausdrücklichen Widerspruchs durch den Patienten - automatisch auf HIV zu testen. In beiden Fällen wurde gegen die "individuelle Suchstrategie" zugunsten einer "gesellschaftlichen Lernstrategie" entschieden und somit die Grundlinie der bundesdeutschen AIDS-Politik beibehalten. Sowohl das Verfügbarwerden von AZT im Jahre 1987 als auch die Ergebnisse, die beim XI. Internationalen AIDS-Kongress in Vancouver im Juli 1996 eine Ära verbesserter therapeutischer Möglichkeiten einläutete, fachten erneute Diskussionen zum Thema HIVAntikörpertestung im Hinblick auf Sekundärprävention an.

\section{Offizielle Politik zur HIV-Antikörpertestung}

\section{Bedeutung des HIV-Antikörpertests im Rahmen des Gesamtkonzepts der HIV- und AIDS-Prävention}

Im Einklang mit den Empfehlungen der Vereinten Nationen, der Weltgesundheitsorganisation und der Europäischen Union setzte die Bundesregierung von Anfang an auf Aufklärung als wichtigste gesundheitspolitische Aufgabe bei der Bekämpfung von AIDS. Dabei war es erklärtes Ziel, in Deutschland ein Netzwerk von Aufklärung, Beratung, Betreuung und Forschung zu schaffen sowie Solidarität und Toleranz für die von HIV und AIDS betroffenen Menschen zu wecken. Trotz anfänglicher Einwendungen - speziell aus Bayern wurde für seuchenrechtliche Maßnahmen plädiert - sowie wiederholter Infragestellung dieser Strategie aus aktuellen Anlässen wurde dieses Ziel bis heute beibehalten. Wesentli- 
cher Bestandteil des Konzeptes war ein Appell an alle Bürger, sich nach definierten Risikosituationen freiwillig - und wenn gewünscht anonym - testen zu lassen. Dabei wurde die Notwendigkeit einer eingehenden Beratung vor und nach Testdurchführung hervorgehoben.

Im Rahmen des bundesdeutschen Gesamtkonzepts zur Bekämpfung von HIV und AIDS hatte der HIV-Antikörpertest neben der Aufklärung und Beratung eher eine untergeordnete Bedeutung; eine auf den Test fokussierte Empfehlung konnte bei einer Auswertung der unterschiedlichsten Informationsbroschüren nicht gefunden werden. $\mathrm{Ob}$ und wenn ja unter welchen Bedingungen die Durchführung des HIV-Antikörpertestes und die Kenntnis des Antikörperstatus präventive Effekte hat, soll an dieser Stelle nicht diskutiert werden. (Für Regelungen und Verwaltungsvorschriften auf Bundesebene vgl. Anhang.)

\section{"Im Rahmen des bundes- deutschen Gesamtkonzepts zur Bekämpfung von HIV und AIDS hatte der HIV-Antikörpertest neben der Aufklärung und Beratung eher eine untergeordnete Bedeutung."}

Gesundheitspolitische Fragen fallen in der Bundesrepublik Deutschland in das Gebiet der "konkurrierenden Gesetzgebung”, das heißt, den Ländern steht für bestimmte Bereiche Gesetzgebungsbefugnis zu, "solange und soweit" der Bund auf diesen Gebieten von seinem Gesetzgebungsrecht nicht Gebrauch macht. Auch die Ausführung der Bundesgesetze ist nach Artikel 83 Grundgesetz Aufgabe der Länder. Vor diesem Hintergrund wurden rechtliche Regelungen und Verwaltungsvorschriften, die sich auf HIV bzw. AIDS beziehen, auch auf Länderebene betrachtet (zur Organisationsstruktur der staatlichen, mit AIDS befassten Institutionen vgl. [7], S. 19 ff.).

Während sich die Mehrheit der Bundesländer in der Frage der HIV-Testung dem gesundheitspolitischen Gesamtkonzept der Bundesregierung angeschlossen und dies auch in entsprechenden Regelungen auf Länderebene bekundet hat, haben einige Bundesländer ergänzende bzw. abweichende Er- klärungen und Vorschriften erlassen. Dies insbesondere im Hinblick auf die Untersuchung von Strafvollzugsgefangenen und Asylbewerbern, zwei Gruppen, die in einem besonderen Abhängigkeitsverhältnis zum Staat stehen.

Besonders bemerkenswert sind darüber hinaus bayerische Vollzugshinweise (siehe oben) zur Durchführung von HIV-Antikörpertests bei i. V.-Drogensüchtigen, bei denen "die Voraussetzungen eines Ansteckungsverdachtes" nach Bundes-Seuchengesetz grundsätzlich als erfüllt gelten. Deshalb "ist eine Blutentnahme zur Durchführung eines HIV-Antikörpertestes erforderlich. Ist der Untersuchungsbefund negativ, gehört jedoch der/die Betroffene weiter dem Kreis der Ansteckungsverdächtigen an, so ordnet die Kreisverwaltungsbehörde an, dass die Untersuchung in vierteljährlichen Abständen wiederholt wird.” Als weitere Besonderheit sollte in diesem Zusammenhang die Anweisung des bayerischen Innenministeriums vom Juni 1987 erwähnt werden, Beamtenanwärter im Rahmen der Einstellungsuntersuchung auf HIV zu testen. Seit November 1995 werden gemäß Ministerratsbeschluss diese obligatorischen HIV-Antikörpertests nicht mehr durchgeführt. Die Gründe hierfür dürften in erster Linie finanzieller Art gewesen sein, da mit dieser Maßnahme kein einziger HIV-infizierter Beamtenanwärter identifiziert werden konnte.

\section{Zustimmung zum HIV-Antikörper- test/Freiwilligkeit}

In Deutschland ist - mit Ausnahme der Blutspenden im Allgemeinen und ausgewählten Situationen in Bayern (vgl. unten) - ausschließlich die freiwillige, auf Wunsch auch anonyme Testung erlaubt. Dabei ist die Beratung vor und nach Testung sowie die ausdrückliche Einwilligung vorgeschrieben (informed consent); alles andere verstößt gegen Prinzipien der ärztlichen Ethik und wäre auch straf- und zivilrechtlich fragwürdig (Körperverletzung bzw. schwere Körperverletzung nach $\$ 223$ a Strafgesetzbuch). Zwar muss davon ausgegangen werden, dass diese Vorschriften nicht immer eingehalten werden, dies steht jedoch eindeutig im Widerspruch zur offiziellen Linie und wird - wo bekannt - geahndet und eingestellt. Ver- schiedene Gerichtsurteile bestätigen dies auch für Einstellungsuntersuchungen, Untersuchungen während der Schwangerschaft und bei der Krankenhausaufnahme.

Im Falle der Ablehnung eines freiwilligen, jedoch dringend angeratenen HIV-Antikörpertestes von "Personen aus der Gruppe definierter Hauptgefährdeter" (nach schriftlicher und mündlicher Belehrung) wird im Justizvollzugsbereich in Ausnahmefällen (Schleswig-Holstein [8]) das Angebot wiederholt, in anderen Fällen wird der Häftling behandelt, als sei er HIV-positiv (Saarland [9]). In Bayern ist die Möglichkeit der Ablehnung gar nicht vorgesehen.

\section{Verfügbarkeit des HIV- Antikörpertests}

In Deutschland ist der HIV-Antikörpertest flächendeckend bei unterschiedlichen Stellen zu erhalten. Jeder Arzt, unabhängig davon, ob in eigener Praxis oder im Klinikbereich, ist im Rahmen differentialdiagnostischer Erwägungen berechtigt, den HIV-Antikörpertest auf Kosten der Krankenkassen durchzuführen. Wenn keine derartigen Gründe vorliegen, kann er jederzeit auf Wunsch und Kosten des Patienten einen HIV-Antikörpertest veranlassen. Viele Gesundheitsämter und spezielle AIDS-Beratungsstellen bieten die Möglichkeit des anonymen und kostenlosen HIV-Antikörpertestes. Darüber hinaus führen auch einige Laboratorien gegen Bezahlung direkt (dort allerdings in der Regel ohne Beratung) eine Blutuntersuchung auf Antikörper gegen HIV durch.

\section{"Pro Jahr werden in Deutschland schätzungsweise (ohne Blutspenden) etwa 2 Millionen HIV-Tests durch- geführt, dabei werden ca. 2000 neue HIV-Infektionen entdeckt."}

Das niedrigschwelligste Angebot dürfte das bei den anonymen Beratungsstellen der Gesundheitsämter sein. Da es sowohl kostenlos als auch anonym zur Verfügung steht, sind evtl. objektive Hinderungsgründe allenfalls in einer schlechten Erreichbarkeit z. B. im ländlichen Bereich zu vermuten. 


\section{Testhäufigkeit/Anteil positiver Ergebnisse}

Da in Deutschland nur die Bestätigungstests im Rahmen der Laborberichtsverordnung meldepflichtig sind, gibt es über die Gesamtzahl durchgeführter HIV-Tests - außer für spezielle Bereiche, z. B. das Blutspendewesen - keine genauen Angaben. Eigene Erhebung bei allen deutschen Laboratorien zur Gesamtzahl ihrer Suchtests für jeweils einen ausgewählten Monat ergaben auf das Jahr hochgerechnet für das Jahr 1992 eine Gesamtzahl von ca. 2,2 Mio., für das Jahr 1999 [10] eine Gesamtzahl von ca. 2 Mio. (ohne Blutspenden und AUT). Bezogen auf die jährlich an das HIV-Register eingereichten Berichte über "Neuinfektionen" von ca. 2000 ergibt sich ein Anteil von etwa $1 \%$ positiver Testergebnisse. $\mathrm{Zu}$ berücksichtigen ist dabei, dass die Befragung nur einen Rücklauf von ca. $85 \%$ hatte, und - da es sich beim deutschen HIV-Register um testbezogene Meldungen handelt - möglicherweise nicht alle Neuinfektionen als solche erfasst sind. Dennoch dürfte der ermittelte Anteil als grobe Schätzung seinen Zweck erfüllen.

\section{Grundsätzliche Ablehnung einer Routinetestung auf HIV}

Es wird immer wieder ausdrücklich darauf hingewiesen, dass Routinetestungen weder im Gesundheitswesen (in Fällen medizinischer Indikation zum Test ist entsprechend der geltenden Rechtslage die Einwilligung des Patienten einzuholen), noch bei Einstellungsuntersuchungen bzw. im Strafvollzug oder bei Einreise und Aufenthalt von Ausländern in der Bundesrepublik ein geeignetes Mittel zur AIDS-Prävention sein können ([9]; dort sind auch alle Empfehlungen unter "Anhang-Dokumente" sowie eine "Chronik der Ereignisse" abgedruckt).Zwangsmaßnahmen wurden nur in Ausnahmefällen ("Fälle sozial unverträglichen Verhaltens der Unbelehrbarkeit, in denen Infizierte oder Ansteckungsverdächtige aus bewusster Gefährdungsabsicht trotz eingehender Beratung und angebotener Hilfen andere einem Infektionsrisiko aussetzen") als geeignete Mittel der AIDS-Verhütung und -bekämpfung angesehen.

\section{Partnerbenachrichtigung}

Bei der Mitteilung eines positiven Testergebnisses sowie in den entsprechenden printmedialen Informationen für HIVPositive im Hinblick auf künftiges Verhalten wird stets darauf hingewiesen, dass - neben den behandelnden Ärzten auch die jeweiligen Sexualpartner von der Infektion in Kenntnis gesetzt werden sollten bzw. alles Nötige zu deren Schutz unternommen werden muss. Das vor allem in angelsächsischen Ländern diskutierte und teilweise praktizierte kostenund personalaufwendige Verfahren der "partner notification" durch den öffentlichen Gesundheitsdienst ist in Deutschland unüblich und auch im Zusammenhang mit der HIV-Infektion nie ernsthaft in Erwägung gezogen worden. Die Deutsche Krankenhausgesellschaft hat 1988 gemeinsam mit der Bundesärztekammer jedoch eine Empfehlung vorgelegt, in der u. a. zur Frage der Schweigepflicht Stellung genommen wird: "Über dem Vertrauensverhältnis zwischen dem HIV-infizierten Patienten und seinem Arzt stehen als höhere Rechtsgüter Leben und Gesundheit. Das bedeutet, dass der Arzt gefährdete Personen (z. B. wenn der infizierte Patient seinem Partner die Infektion $\mathrm{zu}$ verschweigen gedenkt) in diesen Fällen warnen darf. Eine Verpflichtung zur Warnung des Partners des HIV-infizierten Patienten besteht dann, wenn auch der Partner des Infizierten bei demselben Arzt in Behandlung ist" [11].

Am 5. Oktober 1999 schrieb das Oberlandesgericht Frankfurt am Main (Urteil Az: 8U 7/99) dem Schutz des Lebens und der Gesundheit eines konkret von einer Ansteckung mit HIV bedrohten Patienten Vorrang vor dem ärztlichen Schweigegebot zu und bestätigte damit letztlich die oben zitierte Empfehlung. Hintergrund des Urteils war die Schmerzensgeldklage einer Frau, die - wie ihr HIV-infizierter Lebensgefährte - in der Behandlung desselben Arztes war, der seine Patientin nicht über die Infektion ihres Partners unterrichtet hatte.

\section{HIV-Testpolitik/-praxis nach Bevölkerungsgruppen und Situationen}

Die spezielle Testpolitik und -praxis stellt sich in verschiedenen Bevölkerungsgruppen und Situationen durchaus unterschiedlich dar:

\section{Männer, die Sex mit Männern haben}

Wegen der mangelnden therapeutischen Konsequenzen bei gleichzeitig vorhandener Gefahr von weitreichenden psychischen Folgen nach Mitteilung eines positiven Testergebnisses war der HIVAntikörpertest in den 8oer Jahren gerade unter den AIDS-Hilfen und homosexuellen Selbsthilfegruppen sehr umstritten. Spätestens seit Anfang der goer Jahre hat sich durch Möglichkeiten der Prophylaxe von opportunistischen Infektionen (OI-Prophylaxe) und die krankheitsverzögernden Effekte des therapeutischen Einsatzes von AZT und DDI und später auch von Zwei- und Dreifach-Kombinationstherapien vor allem die Position der AIDS-Hilfen im Hinblick auf den HIV-Antikörpertest sehr verändert. Im "Positionspapier des Vorstandes der Deutschen AIDS-Hilfe zum Test" von 1992 [12] wird konstatiert: "Der HIV-Antikörpertest hat in der Hauptbetroffenengruppe der schwulen Männer eine hohe Akzeptanz erfahren. .... Die Prophylaxemöglichkeiten... können $\mathrm{zu}$ mehr Lebensqualität führen. Darum rät die Deutsche AIDS-Hilfe Menschen aus den Hauptbetroffenengruppen mit realem Infektionsrisiko, ihre möglicherweise ablehnende Haltung zum Test zu überdenken." Im Jahre 1997 wird von den Behörden und dem Gesetzgeber nachdrücklich gefordert, dass entsprechende Rahmenbedingungen geschaffen werden, u. a. durch Sicherstellung einer umfassenden und persönlichen Beratung sowie "die strafrechtliche Verfolgung unfreiwilliger oder leichtfertig herbeigeredeter Testungen" [13]. Diese Haltung ist noch immer unverändert. Die Deutsche AIDS-Hilfe (D.A.H.) zählt in ihrer aktuellen Broschüre zum HIV-Test eine Reihe von “vernünftigen Gründen" für den Test auf, rät jedoch weiterhin zu einer gründlichen Abwägung in einem Beratungsgespräch [14].

\section{Ausländer/Asylanten}

Votum 6 des Nationalen AIDS-Beirates zur HIV-Antikörpertestung von Ausländern (29.9.1987[15]): "Der Arbeitsgemeinschaft der Medizinalbeamten folgend, wird festgestellt, daß durch HIVAntikörpertests bei allen Ausländern kein wirksamer Beitrag zur Seuchenbe- 
kämpfung geleistet werden kann.” In den bayerischen Vollzugshinweisen [16] wird die Erteilung der Aufenthaltsgenehmigung für mehr als drei Monate an Ausländer, die nicht aus westeuropäischen Staaten bzw. der EG kommen sowie die Gewährung von Asyl für Bewerber aus außereuropäischen Ländern und der Türkei, von der Durchführung eines HIVTests abhängig gemacht. Seit September 1994 wird mittels einer Änderung der Bekanntmachung ein HIV-Antikörpertest im Rahmen der ärztlichen Untersuchung für die Erteilung einer Aufenthaltsbewilligung nur noch bei Personen, die aus Staaten mit einer hohen HIV-Prävalenz kommen, für erforderlich gehalten. Eine entsprechende Länderliste wird in regelmäßigen Abständen aktualisiert.

Eine Nachfrage nach dem weiteren Procedere im Falle eines positiven HIVTests hat ergeben, dass der HIV-positive Asylbewerber durch Unterschrift bestätigen muss, dass er über seine Infektion aufgeklärt und seinerseits angewiesen wurde, seine Sexualpartner und behandelnden Ärzte von seiner Infektion in Kenntnis zu setzen. Bei entsprechend fortgeschrittenem Immundefekt wird sowohl eine medizinische als auch psychologische Betreuung in die Wege geleitet (telefonische Auskunft 6/2000).

Auch in Sachsen ist laut einer gemeinsamen Vorschrift der Gesundheitsämter aus dem Jahre 1992 [17] "bei jedem Asylbewerber ab dem 14. Lebensjahr nach entsprechender Information ein HIV-Antikörpertest durchzuführen”.

\section{"Ein positiver HIV-Test kann für Nicht-EU-Bürger die Verweigerung des Aufenthaltsrechts und eines Ausbildungsstipendiums zur Folge haben."}

Dagegen wird zur Frage der Untersuchung auf HIV-Antikörper bei Asylbewerbern in einem Erlass von 1993 in Mecklenburg-Vorpommern [18] ausdrücklich festgelegt, dass allen Asylbewerbern die Möglichkeit des anonymen HIV-Antikörpertestes angeboten werden soll. Hierbei sollen Testempfehlungen in 17 Sprachen (!) behilflich sein. In diesen wird dem Asylbewerber u. a. versichert, dass "das Ergebnis der Untersuchung keine Auswirkung auf die Aner- kennung" des Asylantrages hat. Der Asylbewerber muss sein Einverständnis zur Testung durch Unterschrift erklären.

Langzeitstipendiaten der Deutschen Stiftung für internationale Entwicklung, die aus Entwicklungsländern stammen, benötigen ein Gesundheitszeugnis, einschließlich negativem HIVAntikörpertest. Der Test muss einmal im Herkunftsland und ein zweites Mal in der Bundesrepublik durchgeführt werden. "Wird eine Infektion festgestellt oder ist der Teilnehmer aus anderen Gründen gesundheitlich für die Fortbildung nicht geeignet, ist er verpflichtet, unverzüglich in sein Heimatland zurückzukehren" [19]. Diese Praxis wurde vor ca. einem Jahr geändert. Seither ist lediglich ein HIV-Antikörpertest im Heimatland vorgeschrieben; nur seronegative Bewerber werden bei der Auswahl berücksichtigt (telefonische Auskunft).

\section{Ausland (Arbeit im Ausland/Reisen ins Ausland)}

Votum 3 des Nationalen AIDS-Beirates zur HIV-Antikörpertestung bei Reisetätigkeit (5.3.1987): “Das Risiko einer berufsbedingten HIV-Infektion ist äußerst gering. Eine routinemäßige AntikörperTestung im Zusammenhang mit einer Dienstreise ist nicht notwendig. Reisende in Endemie-Gebiete sollten über Infektionsrisiken aufgeklärt werden.” Votum 10 des Nationalen AIDS-Beirates zu HIV-Infektion und Tropentauglichkeitsuntersuchungen (16.12.1987) stellt zwar fest, dass ein HIV-Antikörper-Test "nicht obligat Bestandteil der Tropentauglichkeits- und Rückkehreruntersuchungen ist", führt jedoch weiter aus: "Bei anamnestischen oder klinischen Hinweisen auf Vorliegen einer HIV-Infektion wird der HIV-Test dringend empfohlen. Aufklärung und Einverständnis des Patienten sind hierzu erforderlich. Wird in diesem Falle die Untersuchung abgelehnt, erfolgt die Beurteilung: 'Gegen den vorgesehenen Aufenthalt bestehen zeitlich befristete Bedenken, weitere ärztliche Abklärung erforderlich'. Bei positivem Testergebnis ohne weitere Hinweise auf eine Immundefizienz erfolgt die Beurteilung: 'Gegen den vorgesehenen Aufenthalt bestehen keine Bedenken unter der Voraussetzung, dass eine ärztliche Verfügbarkeit gewährleistet ist und eine Kontrolluntersuchung nach etwa einem Jahr durchgeführt werden kann.' Erge- ben sich Hinweise auf Immundefizienz, erfolgt die Beurteilung: 'Gegen den vorgesehenen Aufenthalt bestehen dauernde Bedenken." Votum 28 des Nationalen AIDS-Beirates zu AIDS-Prävention und Reisen (22.6.1991) betont die Notwendigkeit der Förderung von präventionsgerechtem Verhalten für alle Urlaubsreisenden, Dienst- und Geschäftsreisende sowie Menschen, die sich berufsbedingt längere Zeit im Ausland aufhalten. Er empfiehlt den konsequenten Gebrauch von Kondomen und fordert alle Reiseanbieter und -veranstalter sowie alle Institutionen/Firmen, die Mitarbeiter ins Ausland entsenden, aber auch Ärzte, die Reisende gesundheitlich beraten, auf, Aufklärungsarbeit zu leisten.”

In der Praxis sind diese Voten Grundlage für eine mehr oder weniger intensive Testempfehlung, der sich in einigen Fällen auch das Testangebot für die Angehörigen der im Ausland eingesetzten Menschen anschließt. Rücksprachen mit dem Deutschen Entwicklungsdienst, der Deutschen Stiftung für internationale Entwicklung, dem Auswärtigen Amt, der Gesellschaft für Technische Zusammenarbeit haben ergeben, dass auch hier jeweils der Test erst durchgeführt wird, wenn eine ausführliche Beratung erfolgt ist und eine schriftliche Einverständniserklärung des Testwilligen vorliegt.)

In den Fällen, in denen die Partnerländer den HIV-Antikörpertest als zwingende Voraussetzung für die Erteilung eines Einreisevisums bzw. einer Aufenthaltsgenehmigung vorschreiben, werden die zu Untersuchenden aufgefordert, in jedem Falle eine Durchführungsgenehmigung für den HIV-Antikörpertest zu erteilen. Nach Auskunft des ärztlichen Dienstes der Seeberufsgenossenschaft Hamburg können Seeleute, die einen HIV-Antikörpertest wünschen, diesen kostenlos und anonym beim hafenärztlichen Dienst erhalten. Routinemäßige Tests werden auch hier nicht durchgeführt. Der Flughafenärztliche Dienst Düsseldorf - Koordinationsstelle Reisemedizin untersucht regelmäßig fliegendes Personal, aber auch Menschen im Rahmen von Auslandseinsätzen der Industrie und des Handwerks und bietet darüber hinaus Privatreisenden im Rahmen der Impfsprechstunde seinen Service an. Das Beratungsangebot ist hierbei nicht HIV-spezifisch, sondern auf Infektionskrankheiten allge- 
mein ausgerichtet. Wenn ein Test auf HIV-Antikörper durchgeführt werden soll, ist eine schriftliche Einwilligung erforderlich. Dem Untersuchten - speziell aus dem Personalbereich - wird zugesichert, dass ihm keine beruflichen Nachteile entstehen, sollte er sich gegen eine Testung entscheiden [20].

\section{"Lufthansa - Fürsorgepflicht als Vorwand für berufliche Diskriminierung?"}

\begin{abstract}
"Lufthansa", die Deutsche Fluglinie, die seit einigen Jahren eine private Aktiengesellschaft ist, verfolgt eine Politik des obligatorischen HIV-Tests bei Flugpersonal, das auf Auslandsflügen eingesetzt werden soll. Als Vorwand für dieses Vorgehen wird nach unserer Kenntnis die Notwendigkeit der Gelbfieberimpfung genannt. Lufthansa selbst gibt an, dass es dafür "unterschiedliche Gründe" gibt. Die HIV-Antikörpertestung werde ausschließlich bei Neuanwärtern für den Auslandseinsatz - nach Aufklärung und schriftlicher Einverständniserklärung - durchgeführt, es erfolgen keine weiteren regelmäßigen Kontrollen. Im Fall eines positiven Testergebnisses "wird dem Betroffenen bei entsprechender Qualifikation ein Arbeitsplatz beim Bodenpersonal angeboten" (schriftliche Auskunft vom Juni 2000).
\end{abstract}

\section{Intravenöse Drogen-Benutzer}

Votum 12 des Nationalen AIDS-Beirates zu AIDS und Drogen (16.12.1987) stellt fest, "dass das Problem der HIV-Infektion bei Drogenabhängigen durch eine Erweiterung und Intensivierung der derzeitigen Programme für Drogenabhängige, insbesondere durch alternative Angebote im ambulanten und teilstationären Bereich anzugehen ist. ... Die Durchführung eines Antikörper-Testes ist wünschenswert, sie darf aber nicht als Eingangsvoraussetzung für die Therapie festgeschrieben werden."

Anmerkung: Abweichende Regelungen vgl. Bayern

Votum 23 (22.5.1990) bestätigt die Grundaussagen und fordert konkrete, definierte Präventionsmaßnahmen. Auch die Enquete-Kommission des Bundestages hat in ihrem Abschlussbericht ausführlich zum Thema Drogenge- brauch und HIV-Prävention Stellung genommen bzw. konkrete Vorschläge unterbreitet [21].

\section{Krankenhauspatienten}

Eine generelle HIV-Antikörpertestung von Patienten wird als nicht sinnvoll erachtet, weder zum eigenen noch zum Schutz der Beschäftigten im Gesundheitswesen (Ausnahme: Notstandsgesichtspunkt $\$ 34$ Strafgesetzbuch). Auch wenn es medizinisch indiziert ist, kann nicht von einem stillschweigenden Einverständnis zum Test ausgegangen werden."Die Einwilligung in eine Blutentnahme zum Zwecke der Labordiagnostik ist hierfür nicht ausreichend. Eine formularmäßige Einwilligung in den Test im Rahmen der Aufnahmeformalitäten in das Krankenhaus ist nicht ausreichend" [22]. Deutsche Krankenhausgesellschaft und Bundesärztekammer gehen in "Gemeinsamen Empfehlungen" davon aus, dass nur in bestimmten Fällen ausnahmsweise nach eingehender Prüfungvon einerstillschweigendenEinwilligung des Patienten ausgegangen werden kann, nämlich dann "wenn der dem Arzt erteilte Untersuchungsauftrag im Einzelfall bei verständiger Würdigung die Einwilligung umfasst, das abgenommene Blut auch zur Durchführung der HIV-Diagnostik zu verwenden”. Auch in diesen Ausnahmefällen "ist ein behutsam und schonend geführtes Aufklärungsgespräch sinnvoll”.... "Verweigert ein Patient die Durchführung eines HIV-Tests, obwohl dieser aus medizinischer Sicht geboten ist, so ist das Krankenhaus zur Behandlung/Weiterbehandlung grundsätzlich nicht verpflichtet, soweit die Durchführung des Tests notwendige Voraussetzungder Behandlung ist." [11]. Im Votum 15 des Nationalen AIDS-Beirates zur HIV-Antikörpertestung im Krankenhaus (21.9.1987) werden Bundesärztekammer und Deutsche Krankenhausgesellschaft aufgefordert, diese Haltung zu überprüfen.

In der Praxis werden besonders im chirurgischen Bereich Patienten ab und zu mit dem Problem der HIV-Testung ohne ihre explizite Zustimmung konfrontiert. Diese Praxis führte verschiedene Male zu zivilgerichtlichen Auseinandersetzungen mit unterschiedlichen gerichtlichen Entscheidungen. In einem Fall, in dem das Testergebnis negativ war, wurde entschieden, dass kein Scha- densersatz gezahlt werden musste, weil kein Schmerz zugefügt worden sei.

\section{Personal im Gesundheitsbereich}

Routinetestungen des medizinischen Personals im Rahmen von Einstellungsoder arbeitsmedizinischen Vorsorgeuntersuchungen sind weder vom Gesetzgeber noch von den Berufsgenossenschaften vorgeschrieben. Votum 2 des Nationalen AIDS-Beirates zur Testung medizinischen Personals (5.3.1987):

"Eine routinemäßige HIV-Testung des medizinischen Personals ist nicht erforderlich. Für Mitarbeiter in besonders exponierten Bereichen wird eine Antikörper-Testung bzw. Einfrierung von Serum vor Aufnahme der Tätigkeit empfohlen." Die Kommission des Bundesgesundheitsamtes "Erkennung, Verhütung und Bekämpfung von Krankenhausinfektionen" [23] stellt 1988 fest: "Die Beachtung anerkannter Regeln der Hygiene ist... bei der Behandlung und Pflege aller Patienten unerlässlich. Die Betreuung von HIV-infizierten Patienten stellt an die Hygiene keine höheren Anforderungen, als sie für Krankheiten erforderlich sind, deren Erreger auf gleichen Wegen verbreitet werden (z. B. Hepatitis B)". Die Bundesärztekammer und die Deutsche Krankenhausgesellschaft sehen in den oben genannten "Gemeinsamen Empfehlungen" [11] Einstellungsuntersuchungen auf HIV-Antikörper und entsprechende Wiederholungsuntersuchungen für Personal mit "erhöhter" Infektionsgefährdung vor und stehen damit im Widerspruch zu den Beschlüssen der Konferenzen der für das Gesundheitswesen zuständigen Minister und Senatoren der Länder [7]. Nachdem der Fall eines Zahnarztes in Florida bekannt und öffentlich diskutiert worden war, wurde eine "gemeinsame Empfehlung" des Deutschen Gesundheitsministeriums und der Bundesärztekammer im Juli 1991 herausgegeben. Sie empfahl, dass alle im medizinischen Bereich Beschäftigten, die invasive Eingriffe durchführen und ein Risiko für eine HIV-Infektion hatten oder immer noch haben, einen HIV-Test auf freiwilliger Basis durchführen sollten. Im Falle eines positiven Testergebnisses sollten sie keine invasiven Eingriffe mehr durchführen. Es wurden weder konkrete Regeln erstellt über den Bereich der medizinischen Eingriffe noch über zu befolgende Verfahrensweisen. 
Recherchen bei allen Fachverbänden von medizinischen Disziplinen mit invasiven Methoden einschließlich Bundeszahnärztekammer ergaben nur in drei Fällen Hinweise auf Richtlinien bzw. Empfehlungen im Hinblick auf HIV:

Der Verband Deutscher Betriebsund Werksärzte e. V. , Berufsverband deutscher Arbeitsmediziner stellt seinen Mitgliedern zwei Artikel aus einem Tagungsbericht von 1988 zur Verfügung: In "Aktuelles zur HIV-Testung im Krankenhaus" wird betont, "dass bei strikter Einhaltung der bekannten Hygiene-Vorschriften weder für die Patienten noch für die Krankenhausmitarbeiter ein erhöhtes Ansteckungsrisiko zu besorgen sei.... Deshalb sollten auch alle HygieneAnstrengungen zur Infektionsprophylaxe tatkräftig unterstützt werden.... Dennoch dürfen die hierdurch zu erzielenden Effekte auch nicht überschätzt werden.” Abschließend jedoch wird konstatiert, dass "mit der Durchführung von HIV-Testen.... anstelle höchster Hygiene-Anforderungen, die in der Praxis dann nicht befolgt werden,... eine... risikoorientierte Infektionsprophylaxe auf angemessenem allgemeinen HygieneNiveau durchgeführt werden" könne [24]. Der zweite Aufsatz "Aktuelles zur HIV-Testung in der gewerblichen Wirtschaft" (beim Betriebsarzt) wägt die HIV-Infektion bzw. die Notwendigkeit zur Testung ab unter den Gesichtspunkten

D Tropentauglichkeit (G 35),

D Fahr-, Steuer- und Überwachungstätigkeiten und Arbeiten mit Absturzgefahr (G 25/41),

D Tätigkeiten, die mit einer Exposition gegenüber immunschädigenden Einflüssen einhergehen.

Der Autor kommt zu dem Schluss: Bis zur Entwicklung neuer Gesichtspunkte "bleibt der HIV-Test bei G35 eine wünschenswerte und dringend zu empfehlende, bei G 25 und G 41 eine evtl. ergänzende (zur differentialdiagnostischen Abklärung eines auffälligen neurologischen Bildes) und bei allen anderen Überlegungen eine rein fakultative Maßnahme [25]. In einem Aufsatz in "Der Betriebsarzt” - mit Mitteilungen des Verbandes Deutscher Betriebs- und Werksärzte - Berufsverband Deutscher Arbeitsmediziner - und der Vereinigung Schweizerischer Betriebsärzte wird ab- schließend festgestellt: "Zum Schutz des Personals und zum Schutz der Patienten sollten deshalb die zur Erkennung der HIV-Infektion notwendigen Untersuchungen in das Routineprogramm der arbeitsmedizinischen Vorsorgeuntersuchungen aufgenommen werden. Die sichere und gefahrlose Beschäftigung des Personals kann aber nur gewährleistet werden, wenn die notwendigen Tests nicht nur bei den arbeitsmedizinischen Erst-, sondern auch bei den Nachuntersuchungen regelmäßig vorgenommen werden" [26]. Ausgelöst durch den "Aachener Fall" - ein Herzchirurg soll mehrere seiner Patienten mit dem Hepatitis-B-Virus (HBV) infiziert haben - ist die Diskussion zur Vermeidung nosokomialer Infektionen durch infiziertes Personal im vergangenen Jahr neu entfacht [27].

Auch in den Bereichen Rettungswesen, Wohlfahrtspflege und Fachberufe im Gesundheitswesen konnten über die vorstehenden Empfehlungen hinaus keine speziellen Empfehlungen zur HIV-Infektion ermittelt werden. Einzige Ausnahme, jedoch nicht schriftlich belegt, scheint das Deutsche Rote Kreuz zu sein, das von jedem Mitarbeiter im Rettungsdienst jährlich und nach Exposition sowie von jedem Auslandsdelegierten im Rahmen der Tropentauglichkeitsuntersuchung vor und nach dem Einsatz einen HIV-Antikörpertest fordert (fernmündliche Auskunft). Leider konnte nicht ermittelt werden, welche Konsequenzen aus einem HIV-positiven Testergebnis resultieren.

In unterschiedlichen Verbänden der Nahrungs- und Genussmittelbranche, aber auch im Handwerksbereich (z. B. Friseure) sind in Deutschland bisher keine Empfehlungen oder Richtlinien zur HIV-Antikörpertestung von Beschäftigten erstellt worden.

\section{Strafvollzug}

Votum 26 des Nationalen AIDS-Beirates zu AIDS und Justizvollzug (29.11.1990): "Für den HIV-Antikörpertest gelten die allgemeinen Grundsätze: Es muss sichergestellt sein, dass der Test freiwillig erfolgt. Der Test muss verbunden sein mit einer eingehenden persönlichen Beratung vor der Durchführung und bei Bekanntgabe des Testergebnisses. Das Testergebnis unterliegt der ärztlichen Schweigepflicht; die Unterrichtung des
Anstaltsleiters und ggfs. weiterer Bediensteter sollte grundsätzlich nur mit Zustimmung des Betroffenen erfolgen.”

Sonderregelungen gibt es beispielsweise im Saarland. Hier wird die Notwendigkeit von Aufklärung sowie Freiwilligkeit und Anonymität des HIVAntikörpertestes bei Strafgefangenen hervorgehoben. Darüber hinaus wird die schriftliche Einverständniserklärung zur Testdurchführung gefordert. Einzelhafträume werden zur Regel erklärt, es sei denn, dass der Mitgefangene über die Infektion informiert wird und seine $\mathrm{Zu}$ stimmung erklärt hat. Nichtgetestete sollen laut Erlass wie Positive behandelt werden [9].

Während Thüringen in einer Verwaltungsvorschrift 1992 [28] vergleichbare Regeln vorgibt und in Ausnahmefällen mit Zustimmung des Anstaltsarztes, des Anstaltsleiters und den informierten Mitgefangenen Zusammenlegungen vorsieht, wird in einem Erlass in Sachsen-Anhalt [29] festgelegt, dass in definierten Gruppen der Test "dringend geboten" sei, und die Anstaltsärzte auf diese Gefangenen "intensiv einwirken" sollen, um diese zum Test zu bewegen.

In Bayern wurde die Frage nach der Testung von Gefangenen durch ein Rundschreiben aus dem Jahr 1987 [30] sowie Vollzugshinweise [16] geregelt. Danach sind alle Gefangenen bei Erstaufnahme und bei Entlassung - nach Mitteilung über den Zweck der Blutentnahme - auf HIV-Antikörper im Blut zu untersuchen. Wird die Blutentnahme nicht freiwillig geduldet, ist die Einsicht in die Notwendigkeit der Untersuchung "eindringlich zu vermitteln". Bei Gefangenen aus definierten Risikogruppen ist die zwangsweise Blutentnahme nach $\S$ 101 Strafgesetz zulässig. Gefangene, die den definierten Risikogruppen angehören, gelten bis zum Vorliegen des Ergebnisses eines HIV-Antikörpertestes als positiv.

Die Testpraxis war Thema einer 1996 im Rahmen eines EU-Auftrages durchgeführten schriftlichen Befragung aller Landesjustizbehörden. Danach wird in allen 11 Bundesländern, die geantwortet haben, die Frage nach dem HIV-Status bei Aufnahme in den Justizvollzug gestellt. Der HIV-Antikörpertest ist freiwillig; die Testbereitschaft der neu Inhaftierten liegt zwischen $19 \%$ in Sachsen-Anhalt und $96 \%$ in Bayern. Im Vergleich dazu wird eine Eingangsuntersu- 
chung auf Hepatitiden in den meisten Ländern gar nicht oder nur bei medizinischer Indikation durchgeführt. HIVpositive Testergebnisse lagen zwischen $0,01 \%$ aller Getesteten in Thüringen und $3,62 \%$ in Rheinland-Pfalz. Externe Präventionsangebote existieren laut Auskunft in einigen Ländern "regelmäßig", in anderen sind sie "gewährleistet" oder werden "auf Verlangen” zur Verfügung gestellt. In allen Ländern - außer Schleswig-Holstein - wird der Serostatus der HIV-positiven Gefangenen vertraulich behandelt, in Hessen, Schleswig-Holstein und Nordrhein-Westfalen jedoch nur gegenüber den Mitgefangenen, nicht gegenüber dem Vollzugspersonal.

\section{"Die Testberatung beschränkt sich in Gefängnissen in der Regel auf schriftliches Informations- material, das ausländischen Gefangenen in der Regel nicht zur Verfügung steht."}

Im Rahmen dieser Untersuchung [31] wurde u. a. auch der Frage nach der HIV-Prävention in ausgewählten deutschen Gefängnissen nachgegangen. Dabei zeigten sich deutliche Unterschiede sowohl zwischen den einzelnen Bundesländern als auch zwischen den unterschiedlichen Gefängnissen. Die Zustimmung des Gefangenen zum Test wird immer (einschließlich Bayern) gefordert. In einigen Bundesländern findet der HIV-Antikörpertest im Rahmen der Aufnahmeuntersuchung statt, in anderen nur auf Nachfrage des Gefangenen. In den östlichen Bundesländern ist der Anteil der HIV-Antikörpertests bei Neuaufnahme deutlich niedriger als in den westlichen Bundesländern. In den meisten Bundesländern wird bei Entlassung kein erneuter HIV-Antikörpertest durchgeführt, so dass keine Aussagen über Serokonversionen im Gefängnis möglich sind. Die Beratung beschränkt sich im Gefängnis in der Regel auf schriftliches Informationsmaterial, aber auch das ist nicht in allen Gefängnissen vorhanden. Für ausländische Gefangene steht in der Regel kein Informationsmaterial zur Verfügung. Die Testmitteilung erfolgt in den meisten Fällen durch den Gefängnisarzt, in einigen Gefängnissen erfolgt eine Nachberatung nur in den Fällen eines Antikörpernachweises. Die
Untersuchung hält die Testberatung in den deutschen Gefängnissen insgesamt für mangelhaft und erklärt dies $\mathrm{z}$. T. durch mangelhaftes Know-how und fehlende Zeit der Ärzte.

Im Falle des Antikörpernachweises bei einem Gefangenen folgen Restriktionen, die je nach Bundesland unterschiedlich sind. Üblicherweise darf der HIV-Positive bestimmte Arbeiten, wie Küchenarbeit oder Friseurarbeiten, nicht verrichten; er wird außerdem in einer Einzelzelle untergebracht. Er darf die Zelle nur mit einem Mitgefangenen teilen, den er über seine gesundheitliche Situation in Kenntnis gesetzt hat. Auf der anderen Seite erhalten HIV-positive Gefangene eine spezielle Diät, die Milchprodukte und Früchte beinhaltet. Sie werden üblicherweise auch von Mitgliedern der lokalen AIDS-Hilfe besucht. Da nicht alle Bundesländer über Vollzugskliniken verfügen, gestaltet sich auch die Therapie von HIV-Positiven völlig unterschiedlich. In Nordrhein-Westfalen werden die Häftlinge zu regelmäßigen Untersuchungen bzw. für therapeutische Belange aus dem Vollzug zur Klinik gebracht.

Eine Untersuchung zur AIDS-Prävention im sächsischen Justizvollzug [32] kommt 1999 zu dem Ergebnis, dass die Testpraxis in Sachsen der anderer Bundesländer entspricht und wertet sie als allgemein fragwürdig. Im Widerspruch zur offiziellen Politik würden die Gefangenen oftmals von Ärzten zum Test genötigt, die geforderte umfassende Testberatung erfolge speziell bei ausländischen Gefangenen gar nicht, in anderen Fällen nicht ausreichend. Auch die ärztliche Schweigepflicht werde nicht in der vom sächsischen Justizministerium vertretenen Weise eingehalten. Die Studie zeigt zwei Lösungsmöglichkeiten auf: Die Freiwilligkeit des Tests sollte durch eine Entkoppelung von der Eingangsuntersuchung gefördert werden, und zur Wahrung der Anonymität sollten externe AIDS-Berater zugezogen werden.

\section{Versicherungsnehmer}

Bei Eintritt in eine gesetzliche Krankenkasse ist ein HIV-Antikörpertest nie, bei privaten Krankenversicherungen "in der Regel nicht” obligatorisch. Durch die Vertragsfreiheit ist es den privaten Krankenversicherungsträgern möglich, in ihren Versicherungsverträgen die
Aufnahme von HIV-Infizierten und AIDS-Kranken auszuschließen bzw. festzulegen, dass die Abgabe wissentlich unrichtiger Angaben zur Person (Nichtangabe einer bekannten HIV-Infektion) zu Leistungsausschluss führt.

Der Hauptausschuss des damaligen Verbands der Lebensversicherungsunternehmen e. V. entschied Anfang 1988, für alle Lebensversicherungsverträge eine Frage nach einem positiven AIDSTest und für alle Verträge über DM 250000 einen HIV-Antikörpertest zusammen mit der ohnehin fälligen ärztlichen Untersuchung zu empfehlen. Im Falle einer vorliegenden Infektion ist der Antragsteller vom Vertragsabschluss ausgenommen. Die Einführung dieser Maßnahme wurde damit begründet, "Schaden durch bewusste Antiselektion von Angehörigen der bekannten Risikogruppen" von ihren Versicherten fernzuhalten [33].

\section{Militärpersonal}

Votum 5 des Nationalen AIDS-Beirates $\mathrm{zu}$ Wehrdienst und HIV-Infektion (29.9.1987):

"Für eine Untersuchung aller Wehrpflichtigen besteht kein Grund. Aufgrund der besonderen Fürsorgepflicht des Bundes wird aber vorgeschlagen, Untersuchungen auf eine HIV-Infektion auf freiwilliger Basis anzubieten. Mit der Untersuchung muss eine Beratung verbunden sein."

Laut Weisung des Verteidigungsministeriums (nicht zugänglich) wird allen Rekruten im Rahmen ihrer Musterung ein HIV-Antikörpertest angeboten. Auch jeder andere Bundeswehrangehörige kann auf Wunsch auf HIV-Antikörper getestet werden. Der Test findet grundsätzlich freiwillig nach entsprechenden Informationsveranstaltungen statt. HIV-positiven Soldaten stehen in den Krankenhäusern der Bundeswehr alle therapeutischen Möglichkeiten zur Verfügung. Nach Auskunft verschiedener Ärzte bei der Bundeswehr werden Test- und gegebenenfalls die Behandlung ohne Druck und Repressalien ausgeübt [21].

\section{Blutspender, Organ- und Samenspender}

Neben der Spenderauswahl [5], die ärztliche Anamnese, Spenderbefragung und 
Selbstausschlussmöglichkeit zum Inhalt hat, wird die Testung aller Blutspenden im Hinblick auf Virussicherheit (auch HIV) ständig aktualisiert. Der seit 1993 am Robert Koch-Institut angesiedelte "Arbeitskreis Blut" berät in allen Fragen der Sicherheit und Effizienz bei der Herstellung, Anwendung und Überwachung von Blut und Blutprodukten. Die Empfehlungen des Arbeitskreises werden in Form von "Voten" allgemein zugänglich gemacht [34]. Seit dem 7. Juli 1998 wird die ordnungsgemäße Gewinnung von Blut und Blutprodukten sowie die sichere Anwendung von Blutprodukten in Deutschland erstmals durch ein Transfusionsgesetz [6] geregelt.

Seit dem 5. November 1997 ist im Rahmen des Transplantationsgesetzes [35] festgelegt, dass "insbesondere das Risiko der Übertragung von Krankheiten so gering wie möglich zu halten" ist. Eine explizite Benennung einzelner Krankheiten ist nicht erfolgt. Jedoch wird in verschiedenen Richtlinien das weitere Prozedere festgelegt. So darf nach den Richtlinien zur Transplantation von Stammzellen aus Nabelschnurblut [36] eine Transplantation nur erfolgen, nachdem $u$. a. eine HIV1/2-Infektion ausgeschlossen wurde.

"Von der Knochenspende auf Dauer auszuschließen sind Spender... bei denen eine HIV-Infektion nachgewiesen wurde", aber auch solche, "die einer Gruppe mit einem gegenüber der Allgemeinbevölkerung deutlich erhöhten Risiko für eine... HIV-Infektion angehören, angehört haben oder dieser zugeordnet werden müssen" [37].

Im Rahmen der Voruntersuchungen zur homologen Insemination müssen HIV-Antikörper-Untersuchungen bei Mann und Frau durchgeführt werden [38]. Darüber hinaus wurde in einer Richtlinie zur Auswahl von Spermaspendern [39] festgelegt, dass der Samenspender vor der ersten Spende und danach alle sechs Monate einen HIVAntikörpertest durchführen lassen muss. "Die donogene Insemination darf nur mit kryokonserviertem Sperma erfolgen, das über eine Quarantänezeit von mindestens 180 Tagen gelagert wurde, wenn der Spender auch nach Ablauf der Quarantänezeit frei von HIV-Antikörpern geblieben ist.” Männer, die Risikogruppen für übertragbare Krankheiten angehören, dürften keine Samenspender sein.

\section{HIV-Antikörpertest im Lichte verbesserter therapeutischer Möglichkeiten}

Jeweils im Sommer 1998 und 2000 wurden Interviews zur Frage der Testempfehlung mit Experten aus unterschiedlichen staatlichen Institutionen, einer sozialwissenschaftlichen Forschungseinrichtung sowie einer NGO (non governmental organization) geführt.

\section{"Die Frage einer generellen Testempfehlung wird im Zusammenhang mit der Sekundärprävention kontrovers diskutiert."}

Grundsätzlich kann festgestellt werden, dass AIDS in der öffentlichen und individuellen Aufmerksamkeit sinkt. Während Mitte der achtziger Jahre AIDS innerhalb der individuellen und politischen Risikowahrnehmung zeitweise das wichtigste Gesundheitsproblem überhaupt zu sein schien, nimmt es politisch heute nur noch einen unteren Rangplatz ein. Die Politik der Primärprävention gegen HIV und AIDS hat sich - auch nach Einschätzung der EnqueteKommission des Deutschen Bundestages - bewährt [21] und wird derzeit nicht in Frage gestellt. Die Frage nach der Notwendigkeit einer generellen Testempfehlung wird im Zusammenhang mit der Sekundärprävention jedoch immer wieder kontrovers diskutiert.

"Die divergierenden Interessenlagen (zur Frage des HIV-Antikörpertests) der letztlich meist von Kontroll-Absichten geleiteten Testbefürworter und der potentiell von der Infektion Betroffenen würden... dann zusammenfallen, wenn durch medizinische Mittel die Progression der Infektion zur Krankheit und/oder der Verlauf der Krankheit und/oder der Lebensdauer günstig beeinflusst werden könnte. In diesem Falle wäre der HIVTest die Eintrittspforte zu nützlicher medizinischer Frühintervention." [40]. In dieser Situation befinden wir uns nach Einschätzung einer Reihe von Experten seit einigen Jahren. Nach Einschätzung der befragten Experten reicht die Verbesserung der Therapiemöglichkeiten jedoch bisher nicht aus, um die generelle Testpolitik zum jetzigen Zeitpunkt zu ändern. Nach allgemeinen Debatten zu diesem Thema ist weder seitens der Bundeszentrale für gesundheitliche Aufklärung noch seitens der Deutschen AIDS-Hilfe eine veränderte Testpolitik in Sicht. Man ist sich einig, dass in Verbindung mit Beratung zur Prävention auch Hinweise auf mögliche Therapieverfahren aufgezeigt werden müssen. Selbstverständlich soll bei der Testmitteilung eines Antikörpernachweises auch ein Schwerpunkt der Beratung auf Behandlungsmöglichkeiten gelegt werden. Es darf jedoch auch nicht verkannt werden, dass z. B. die Notwendigkeit zu lebenslanger Medikation, die Gefahr der Resistenzentwicklung, der Nebenwirkungen und unerwünschten Langzeiteffekte bei frühzeitigem Therapiebeginn eine generelle Testempfehlung nach wie vor nicht nahelegt. Welches der beste Zeitpunkt für den - frühen - Behandlungsbeginn ist, konnte bisher nicht erwiesen werden. Bei nicht antiretroviral vorbehandelten Patienten sind die opportunistischen Infektionen (OI) bei eingeschränktem Immunsystem in der Regel gut therapierbar, auch wenn nach allgemeinem Konsens ein Therapiestart vor der ersten OI als sinnvoll betrachtet wird. Das Für und Wider eines frühzeitigen Behandlungsbeginns wird ausführlich in den Therapierichtlinien [41] diskutiert. Einig sind sich die Experten darin, dass bei entsprechend verbesserten Therapien mehr Menschen mit Risiko bereit sein werden, sich testen zu lassen. Eine gezielte Empfehlung wird mehrheitlich befürwortet, eine generelle Testempfehlung jedoch weiterhin abgelehnt. Eine Zwangstestung steht im Hinblick auf fehlende Effizienz und wegen des unerwünschten Eingriffs in Bürgerrechte weiterhin außer Diskussion.

Es wurden allerdings Befürchtungen laut, dass das Beispiel USA z.B. in bezug auf Schwangere bzw. Neugeborene Schule machen könnte. Dort werden im Zuge verbesserter Möglichkeiten, die Transmissionsrate von HIV-positiven Müttern auf ihre ungeborenen Kinder $\mathrm{zu}$ senken, Schwangere quasi zwangsweise getestet, ohne dass sichergestellt ist, wie ihre eigene medizinische Versorgung ermöglicht werden kann.

Für Deutschland wird nachdrücklich bedauert, dass die in die Mutterschaftsrichtlinien aufgenommene Empfehlung eines Testangebots mit Beratung und entsprechender Anamneseerhebung von den Schwangerschaften betreuen- 
den Ärzten nicht durchgängig befolgt wird. So sehen die Geburtshelfer und Pädiater immer wieder erst perinatal oder im frühen Kindesalter HIV-exponierte oder gar -infizierte Kinder HIVinfizierter Mütter, die während ihrer Schwangerschaft keine Kenntnis über die Infektion hatten. Hier wird vermutlich erst ein Präzedenzfall Abhilfe schaffen, wenn sich eine HIV-infizierte Mutter zur Klage gegen ihren Gynäkologen entschließt, der sie nicht den Mutterschaftsrichtlinien gemäß aufgeklärt hat.

\section{Fazit}

In Anbetracht stagnierender Neuinfektionen darf davon ausgegangen werden, dass sich das von der Bundesrepublik Deutschland verfolgte Gesamtkonzept zur Bekämpfung von HIV und AIDS bewährt hat. Im Rahmen dieses Konzeptes erging ein Appell an alle Bürger, sich nach definierten Risikosituationen freiwillig - und wenn gewünscht anonym - im Zusammenhang mit einer eingehenden Beratung testen zu lassen. Eine allgemeine Testempfehlung oder Vorschriften für Routinetestungen gab es nicht, außer bei Blut-, Organ- und Samenspenden, bei denen die Notwendigkeit einer routinemäßigen Testung unumstritten ist. Gleichzeitig wurde für Solidarität und Toleranz im Umgang mit HIV und AIDS geworben. Die meisten Bundesländer, Verbände und Organisationen haben diese Strategie weitgehend übernommen, einige wenige haben jedoch für verschiedene Bereiche abweichende Regelungen getroffen. Abweichende Regelungen gibt es vor allem für Ausländer und Strafgefangene. Beiden Gruppen werden der sonstigen Bevölkerung vergleichbare Präventionsangebote vorenthalten. Eine Evaluation der Konsequenzen der inkonsistenten AIDS-Politik für diese beiden Personengruppen steht aus.

Durch verbesserte therapeutische Möglichkeiten hat die Frage nach gezielten Testempfehlungen neue Aktualität erlangt.
Anhang

\section{Rechtliche Regelungen und Verwaltungsvorschriften auf der Bundesebene}

Zur Organisationsstruktur der staatlichen mit AIDS befassten Institutionen vgl. [7] S. 19

D Im Regierungsprogramm "Forschung und Entwicklung im Dienst der Gesundheit" des Bundesministeriums für Forschung und Technik wird ein Forschungsschwerpunkt "AIDS" eingerichtet; die Förderziele werden in drei Ausschreibungen (1983, 1985 und 1987) erweitert und differenziert (Bundesministerium für Gesundheit (BMG) 1996).

D Entschließung der Ständigen Konferenz der Kultusminister und -senatoren der Länder "Schule und AIDS" vom 17./18. Oktober 1985 in Köln (BMG 1999, 87-89).

Doalitionsvereinbarung:Maßnahmen zur Bekämpfung von AIDS, März 1987 (BMG 1999, 87).

Dntschließung der Sondersitzung der Konferenz der für das Gesundheitswesen zuständigen Minister und Senatoren der Länder (GMK) vom 27.3.1987 in Bonn (BMG 1999, 89-92).

D Änderung der Richtlinien über die ärztliche Betreuung während der Schwangerschaft und nach der Entbindung (Mutterschafts-Richtlinien) 07/1987.

Dntschließung der 57. Konferenz der für das Gesundheitswesen zuständigen Minister und Senatoren der Länder am 19./20. November 1987 in Osnabrück (BMG 1999, 92-96).

D Die Laborberichtsverordnung legt fest, dass alle Bestätigungstests zum Nachweis von Antikörpern gegen HIV im Form eines anonymen Berichts an das AIDS-Infektionsregister beim Bundesgesundheitsamt zu melden sind. 12/1987 (BGBI. III 2116-1-8-2:3).

Dichtlinien zur ärztlichen Beratung und medizinischen Betreuung bei AIDS und anderen HIV-Infektionen (AIDS-Richtlinien der ehemaligen DDR), 08/1988.

Dntschließung der 59. Konferenz der für das Gesundheitswesen zuständigen Minister und Senatoren der Länder (GMK) am 17./18. November 1988 in Berlin (BMG 1999, 97-98).

D London Declaration on AIDS Prevention vom 23.01.1988. Die Bundesregierung hat sich mit 148 anderen Ländern auf der Gesundheitsministerkonferenz 1988 in London für ein gemeinsames internationales Konzept im Kampf gegen AIDS ausgesprochen. Weitere Vereinbarungen zur internationalen Zusammenarbeit vgl. (BMG 1999, 78-79).

D Endbericht der Enquete-Kommission des 11. Deutschen Bundestages, 1990. Die Einrichtung der Enquete-Kommission "Gefahren von AIDS und wirksame Wege zu ihrer Eindämmung" wurde im Mai 1987 vom Deutschen Bundestag beschlossen. Mitglieder:Neun Abgeordnete aus allen vier Bundestagsfraktionen und acht Sachverständige aus unterschiedlichen Disziplinen (BMG 1999, 20).

Deschluss der Konferenz der Jugendminister und -senatoren der Länder am 19. Mai 1989 (BMG 1999, 100).

D Entschließung der 65. Konferenz der für das Gesundheitswesen zuständigen Minister und Senatoren der Länder (GMK) am 5./6.11.1992 in Schlangenbad (BMG 1999, 98).

Demeinsame Erklärung des Bundesministers für Bildung und Wissenschaft, Bundesministers für Familie und Senioren, Bundesministers für Frauen und Jugend, Bundesministers für Gesundheit anlässlich der Tagung "Jugend und AIDS" am 30.11.1992 (BMG 1999, 101).

Dründung des Fonds "Humanitäre Soforthilfe", aus dem Menschen, die durch Blutprodukte infiziert worden waren, finanzielle Hilfe erhalten sollten 06/1994.

Desetz zur Umstrukturierung des Bundesgesundheitsamtes. Im Robert Koch-Institut, Institut für Infektionserkrankungen und nichtinfektiöse Erkrankungen, AIDS-Zentrum werden weiterhin die Daten zu AIDS und HIV gesammelt und ausgewertet, 06/1994.

Dntschließung der 67. Konferenz der für das Gesundheitswesen zuständigen Minister und Senatoren der Länder am 17./18. November 1994 in Hamburg (BMG 1999, 99).

Das Gesetz über die humanitäre Hilfe für durch Blutprodukte HIV-infizierte Personen (HIV-Hilfegesetz) tritt am 31.07.1005 in Kraft. Eine Stiftung löst den Fonds "Humanitäre Soforthilfe" ab (BMG 1999, 86).

Doten des Nationalen AIDS-Beirates (NAB) 1987-1997 Offizielle Statements zu relevanten Bereichen in Verbindung mit HIV/AIDS (BMG, 1998). Das Bundesgesundheitsministerium hat zur Unterstützung seiner Arbeit ein unabhängiges Beratergremium berufen. Ihm gehören Fachleute aus einschlägigen Berufen an: Ärzte verschiedener Fachgebiete, Pflegekräfte, Psychologen, Sozialarbeiter, Sexualwissenschaftler, Sozialwissenschaftler, Juristen. Ferner sind in dem Gremium Vertreter wichtiger Organisationen vertreten.

D $\$ 20$ Abs.3 SGB V “Selbsthilfeförderung". Die Spitzenverbände der Krankenkassen haben im Interesse einer einheitlichen Rechtsanwendung gemeinsam und einheitlich am 14.02 .1997 beschlossen, HIV und AIDS in das Verzeichnis der Krankheitsbilder aufzunehmen, bei denen die Zusammenarbeit mit Selbsthilfegruppen... mit präventiver oder rehabilitativer Zielsetzung erfolgen kann. 


\section{Literatur}

1. Bundesgesetzblatt (1987) Verordnung über die Berichtspflicht für positive HIV-Bestätigungstests (Laborberichtsverordnung) vom 18.12.1987 BGBI, 1 S.2819). BGBI. III 2116-1-82:3

2. Bales S (2000) Das Infektionsschutzgesetz Zur politischen Neuorganisation der Prävention und Bekämpfung von Infektionskrankheiten in Deutschland. Dissertationsmanuskript. Berlin, S 72-73

3. Czeschinski P (1998) Die Virushepatitiden - Infektionsgefährdung in Beruf und Umwelt.DUV Wiesbaden. S 180

4. Bundesinstitut für Arzneimittel und Medizinprodukte (BfArM) im Bundesgesundheitsamt (20.2.1985) Anordnung:spätestens ab dem 1.10.1985 sind alle Blutspenden auf HIV-Antikörper zu testen

5. Novellierte "Richtlinien zur Blutgruppenbestimmung und Bluttransfusion (Hämotherapie)" (1996). Bundesgesundhbl 39:468

6. Gesetz zur Regelung des Transfusionswesens (Transfusionsgesetz - TFG) (6.Juli 1998). BGBI. Teil I Nr.42

7. Bundesministerium für Gesundheit (BMG) (1999) AIDS-Bekämpfung in Deutschland, 7 . überarb. Aufl. Bonn

8. Der Justizminister des Landes Schleswig-Holstein (29.6.1990/Änderung 22.4.1996). Erlass "Gesundheitsfürsorge in den Justizvollzugsanstalten, hier:Vorsorgemaßnahmen wegen AIDS"

9. Ministerium der Justiz, Saarland (1. Mai 1978) Gemeinsamer Erlass über die Fachaufsicht über die Gesundheitsfürsorge der Gefangenen und die Anstaltsärzte, hier: AIDS-Prophylaxe in den Justizvollzugsanstalten vom 8.1.1993

10. Robert Koch-Institut (2000) Halbjahresbericht: AIDS- und HIV-Infektionen. Ergebnisse einer Umfrage zu HIV-Untersuchungen in deutschen Laboratorien. Epid Bull Sonderausgabe A2000 (im Druck)

11. Deutsche Krankenhausgesellschaft (Hrsg) (01/1988) Gemeinsame Hinweise und Empfehlungen der Bundesärztekammer (BÄK) und der Deutschen Krankenhausgesellschaft (DKG) zur HIV-Infektion

12. Deutsche AIDS-Hilfe e.V. (D.A.H.) (08/1992) Positionspapier des Vorstands der Deutschen AIDS-Hilfe zum Test

13. Deutsche AIDS-Hilfe e.V.(D.A.H.) (11/1997) Der HIV-Antikörpertest, aus: HIV/AIDS. Heutiger Wissensstand

14. Deutsche AIDS-Hilfe e.V. (2/2000) Will ich es wissen? Informationen und Überlegungen zum HIV-Test

15. Bundesministerium für Gesundheit (Hrsg) (1998) Voten des Nationalen AIDS-Beirates 1987-1997. Bonn

16. Bayerisches Staatsministerium des Inneren (19.05.1987) Vollzugshinweise zum Maßnahmenkatalog zur Verhütung und Bekämpfung der Immunschwächekrankheit AIDS - Vollzug des Seuchenrechts, des Ausländerrechts und des Polizeirechts
17. Der Sozialminister des Landes MecklenburgVorpommern (3.1.1993) Erlass zu “Untersuchungen von Asylbewerbern nach dem Asylverfahrensgesetz §62"

18. Sachsen (25.5.1992) Gemeinsame Verwaltungsvorschrift des Sächsischen Staatsministeriums für Soziales, Gesundheit und Familie und des Sächsischen Staatsministeriums des Innern zur gesundheitlichen Betreuung von Asylbewerbern durch die Gesundheitsämter im Freistaat Sachsen

19. Deutsche Stiftung für internationale Entwicklung (DSE) (1998) Bedingungen für die Teilnahme am beruflichen Fortbildungsprogramm der Regierung der Bundesrepublik Deutschland

20. B.A.D.e.V. - Berufsgenossenschaftlicher Arbeitsmedizinischer und Sicherheitstechnischer Dienst, Koordinationsstelle Reisemedizin, Arbeitsmedizin/Flugmedizin (1998) Information zu sexuell übertragbaren Krankheiten/Einverständniserklärung zum HIV-Test/Tipps für Arbeitgeber und Arbeitnehmer vor beruflichen Auslandsaufenthalten. Düsseldorf

21. Deutscher Bundestag, Referat Öffentlichkeitsarbeit (Hrsg) (1990) AIDS: Fakten und Konsequenzen. Endbericht der Enquète-Kommission des 11. Deutschen Bundestages "Gefahren von AIDS und Wirksame Wege zu Ihrer Eindämmung". Bonn

22. Senatsverwaltung für Gesundheit und Soziales, Berlin (11/1989) Informationsmappe "HIV/AIDS in der Arbeitswelt"

23. Kommission für Krankenhaushygiene und Infektionsprävention des Bundesgesundheitsamtes (3.3.1988) Bekanntmachung "Hygienische Maßnahmen zur Verhütung der Übertragung von HIV im Krankenhaus". Bundesgesundhbl 31:3

24. Chriske HW (1988) Aktuelles zur HIV-Testung im Krankenhaus. In: Florian HJ, Tenkhoff N (Hrsg) Tagungsbericht Verband Deutscher Betriebs- und Werksärzte e.V.

25. Klein U (1988) Aktuelles zur HIV-Testung in der gewerblichen Wirtschaft. In: Florian HJ, Tenkhoff N (Hrsg) Tagungsbericht Verband Deutscher Betriebs- und Werksärzte e.V.

26. Chriske HW, Exner M (1987) Zur Beschäftigung HIV-infizierten Personals im Gesundheitswesen. In: "Der Betriebsarzt" mit Mitteilungen des Verbandes Deutscher Betriebs- und Werksärzte - Berufsverband Deutscher Arbeitsmediziner - und der Vereinigung Schweizerischen Betriebsärzte; Arbeitsmedizin - Sozialmedizin Präventivmedizin: 22

27. Nassauer A (1999) Zur Problematik der nosokomialen Übertragung von HIV. Epid Bull 34:251-253

28. Thüringen (1992) Verwaltungsvorschrift "Blutuntersuchungen auf HIV-Antikörper bei Gefangenen

29. Sachsen-Anhalt (06/1991) Erlass "AIDS im Justizvollzug"

30. Bayerisches Staatsministerium des Inneren (3.4.1987) Richtlinien zur Gesundheitsfürsorge in Justizvollzugsanstalten - Maßnahmen wegen AIDS
31. Weilandt C (05/1998) 2. Annual Report - European Network on HIV/AIDS and Hepatitis prevention in prisons

32. Stiehler M (1999) AIDS- und Hepatitisprävention im sächsischen Justizvollzug. Bundesgesundhbl 42:577-582

33. Akermann S (1998) AIDS und Lebensversicherung in Deutschland - 10 Jahre erfolgreiches Risikomanagement.Versicherungswirtschaft, Karlsruhe; Versicherungsmedizin $50 / 3$

34. Burger R (1997) Arbeitskreis Blut: Aufgaben und Ergebnisse. InfFo. 12:8-12

35. Gesetz über die Spende, Entnahme und Übertragung von Organen (Transplantationsgesetz -TPG).BGBI I,263,5.11.1997

36. Deutsches Ärzteblatt (14.5.1999) Richtlinien zur Transplantation von Stammzellen aus Nabelschnutblut ( $\mathrm{CB}=$ Cord Blood). 96, 19:C914-C920

37. Vilmar K, Bachman KD (1996) Richtlinien zum Führen einer Knochenbank. Deutsches Ärzteblatt (26.8.1996)93,34/35:C1551-1555

38. Bundesausschuss der Ärzte und Krankenkassen (14.8.1990) Richtlinien über ärztliche Maßnahmen zur künstlichen Befruchtung

39. Arbeitskreis für donogene Insemination e.V. des Bundesverbandes Reproduktionsmedzinischer Zentren Deutschlands e.V. (1995) Richtlinie zur Auswahl von Spermaspendern. Act. Dermatol 22:36-37

40. Rosenbrock R (1994) Die Normalisierung von Aids - teils nach vorne, teils zurück. AIDS-Forum D.A.H.Sonderband 10 Jahre Deutsche AIDS-Hilfe

41. Deutsch-Österreichische Richtlinien zur Antiretroviralen Therapie der HIV-Infektion (6/1999) URL:

http://www.rki.de/INFEKT/AIDS_STD/BR_LINI E/BR_LINIE.htm 